\title{
REVISÃo de LITERATURA Características das prescrições no transtorno de déficit de atenção/hiperatividade
}

\author{
Medical prescriptions characteristics in attention \\ deficit/hyperactivity disorder \\ Walter Camargos Jr.', Rodrigo Nicolato²
}

\section{RESUMO}

Objetivo: $O$ trabalho busca conhecer, no atual momento, a realidade das prescrições para o transtorno de déficit de atenção/hiperatividade. Método: Revisão não sistemática dos descritores "transtorno de falta de atenção/ hiperatividade" e "prescrição de medicamentos" e seus similares em inglês. Foram utilizados os seguintes limitadores: publicações de 10 anos até novembro de 2008, idiomas inglês, francês, espanhol e português, nas bases de dados do Medline, SciELO, Lilacs, áreas especializadas e Cochrane. Não foram utilizados os artigos referentes ao uso ilícito e aspectos clínicos em geral. Resultados: Foram tratados 8 itens: sistema de prescrições, características socioculturais, os sistemas de saúde que influenciam as prescrições, tempo de uso das medicações, aumento das prescrições, aspectos em comum, dosagens e prescrição em idades abaixo de 5 anos. Conclusões: Alguns aspectos são preocupantes, como a baixa adesão aos tratamentos medicamentosos, doses médias de metilfenidato relativamente baixas e aumento das prescrições de psicoestimulantes para crianças de até 3 anos. Características sociais e o sistema de saúde utilizado pelo afetado também geram grandes variações na prática clínica. Uma questão interessante é a evidência de que o sistema brasileiro de prescrições não é o mais restritivo.

\section{ABSTRACT}

Objective: The paper seeks to know the reality of the prescriptions for the attention deficit/hyperactivity disorder. Method: Non-systematic review of the descriptors "attention deficit / hyperactivity disorder" and "medication prescriptions", in Portuguese and in English similar. The limiters were: 10 years publishings until November 2008, English, French, Spanish and Portuguese languages, in Medline, SciELO, Lilacs, Specialized Areas and Cochrane data base. The articles concerning the drug illicit use and clinical aspects have not been used. Results: Eight items were dealt with: prescriptions system, socio-cultural characteristics and health systems that have influence on the prescriptions, medication use span, increasing prescriptions, common aspects, dosage and prescription to below 5 years old children. Conclusions: Some aspects are worrying, such as the low adhesion to the medical treatments, relatively low average doses and psychostimulants increasing prescriptions even for children up to 3 years old. Social characteristics and the health system used by the affected ones give origin to great variations on the clinical practice. An interesting issue is the evidence that the Brazilian prescriptions system isn't the most restrictive one.

1 Fundação Hospitalar do Estado de Minas Gerais (FHEMIG), Hospital Infantil João Paulo II, Instituto de Previdência dos Servidores do Estado de Minas Gerais (IPSEMG), Programa de Pós-graduação em Ciências da Saúde. 


\section{INTRODUÇÃO}

O transtorno de déficit de atenção/hiperatividade (TDA/H) tem sido extensamente trabalhado do ponto de vista científico nessas duas últimas décadas. Aspectos genéticos, clínicos, terapêuticos via psicofarmacologia ou psicoterápicos, familiares, pedagógicos ou mesmo jurídicos têm sido propagados através de publicações em revistas e livros, assim como por cursos direcionados a profissionais médicos e não médicos. Partindo-se do pressuposto atualmente aceito de que o TDA/H é de origem neurobiológica e que, portanto, o tratamento psicofarmacológico é essencial, além de ser a menos onerosa das terapêuticas indicadas, seria importante se conhecer como tanto conhecimento produzido reflete na qualidade assistencial cotidiana. As características das prescrições podem demonstrar se os médicos têm incorporado os novos conhecimentos produzidos na prática clínica.

\section{OBJETIVO}

O trabalho busca conhecer a realidade das prescrições para o transtorno de déficit de atenção/hiperatividade (TDA/H) a partir de algumas respostas como: qual o profissional que mais prescreve psicoestimulantes? Qual o tempo médio de tratamento com medicação? Qual é o perfil do paciente que se trata com o psiquiatra? Essa realidade é similar à nossa? Essas informações podem melhorar nossa prática clínica? O presente artigo não tem objetivo clínico propriamente dito.

\section{MÉTODOS}

Pesquisa dos descritores "transtorno de falta de atenção/ hiperatividade" e "prescrição de medicamentos" e seus similares em inglês, por 10 anos até novembro de 2008, utilizando os limitadores dos idiomas inglês, francês, espanhol e português nas bases de dados do Medline, SciELO, Lilacs, áreas especializadas e Cochrane. Não foram utilizados os artigos referentes ao uso ilícito e aspectos clínicos em geral.

\section{RESULTADOS}

Foram utilizados 14 artigos da pesquisa (Quadro 1) e outros 5 das referências, sendo tratados 8 itens: sistema de prescrições; características socioculturais; sistemas de saúde que influenciam as prescrições; tempo de uso das medicações; aumento das prescrições; dosagens; prescrição em idades abaixo de 5 anos; e resultados comuns (Quadro 2).
Quadro 1. Resultado da pesquisa bibliográfica

\begin{tabular}{lccccc}
\hline & Medline & Lilacs & $\begin{array}{c}\text { SciEl0 e } \\
\text { Cochrane }\end{array}$ & $\begin{array}{c}\text { ADOLESC } \\
\text { (áreas especializadas) }\end{array}$ & Referências \\
\hline Total de artigos & 62 & 1 & - & 12 & - \\
$\begin{array}{l}\text { Aspectos clínicos, } \\
\text { comentários, outros* }\end{array}$ & 30 & - & - & 4 & - \\
$\begin{array}{l}\text { Mau uso } \\
\text { da medicação* }\end{array}$ & 10 & - & - & 8 & - \\
$\begin{array}{l}\text { Outras línguas* } \\
\text { Utilizados }\end{array}$ & 9 & - & - & - & - \\
\hline
\end{tabular}

* artigos descartados devido a não especificidade ao tema.

Quadro 2. Resultados comuns

\begin{tabular}{|c|c|c|c|c|}
\hline Autor & Aumento & Sexo & $\begin{array}{c}\text { Aumento dos } \\
\text { psicoestimulantes } \\
\text { de longa ação }\end{array}$ & Prescritores \\
\hline $\begin{array}{l}\text { Frances, } \\
2004\end{array}$ & $\begin{array}{l}1997 \text { a 2001: } \\
250 \%\end{array}$ & si & si & si \\
\hline $\begin{array}{l}\text { Preen, } \\
2008\end{array}$ & si & $\begin{array}{l}\text { Homens: } \\
32 \% \text { mais } \\
\text { tratados }\end{array}$ & + & $\begin{array}{l}\text { Pediatra: } 91,5 \% \\
\text { Psiquiatra } \\
\text { Infantil: } 8,5 \%\end{array}$ \\
\hline $\begin{array}{l}\text { Carlini, } \\
2003\end{array}$ & si & $\mathrm{si}$ & si & $\begin{array}{l}\text { Neurologista: } 79 \% \\
\text { Psiquiatra: } 60 \%\end{array}$ \\
\hline $\begin{array}{l}\text { Truter, } \\
2005\end{array}$ & si & $75 \% \mathrm{M}$ & si & $\begin{array}{l}\text { Primary Care: } 53,5 \% \\
\text { Pediatra: } 18,5 \%\end{array}$ \\
\hline $\begin{array}{l}\text { Castle, } \\
2007\end{array}$ & $\begin{array}{l}2000 \text { a 2005: } \\
11,8 \% \text { do } \\
\text { tratamento }\end{array}$ & $\begin{array}{l}\text { Homens } \\
\text { mais } \\
\text { medicados: } \\
230 \%\end{array}$ & + & \\
\hline $\begin{array}{l}\text { Miller, } \\
2004\end{array}$ & si & $85 \% \mathrm{M}$ & si & $\begin{array}{l}\text { Primary Care: } 55,5 \% \\
\text { Pediatra: } 23 \% \\
\text { Psiquiatra: } 21 \%\end{array}$ \\
\hline $\begin{array}{l}\text { Knellwolf, } \\
2008\end{array}$ & $\begin{array}{l}1997 \text { a 2005: } \\
800 \%\end{array}$ & si & + & si \\
\hline Lin, 2005 & $\begin{array}{l}\text { Prescrições de } \\
\text { anfetaminas: } \\
1990 \text { a } \\
1993: 59 \% \\
1993 \text { a 1997: } \\
187 \%\end{array}$ & si & $\mathrm{Si}$ & si \\
\hline $\begin{array}{l}\text { Miller, } \\
2001\end{array}$ & $\begin{array}{l}\text { Prescrições: } \\
1990 \text { a 1996: } \\
500 \%\end{array}$ & si & $\mathrm{Si}$ & si \\
\hline $\begin{array}{l}\text { McCarthy, } \\
2006\end{array}$ & si & si & + & si \\
\hline $\begin{array}{l}\text { Robison, } \\
1999\end{array}$ & $\begin{array}{l}1990 \text { a 1995: } \\
250 \% \\
\text { diagnósticos } \\
\text { de TDAH }\end{array}$ & $85 \% \mathrm{M}$ & $\mathrm{Si}$ & $\begin{array}{l}\text { Pediatra: } 40 \% \\
\text { Psiquiatra: } 23,5 \\
\text { Primary Care: } 17,5 \%\end{array}$ \\
\hline $\begin{array}{l}\text { Fogelman, } \\
2001\end{array}$ & $\begin{array}{l}1992 \text { a 1999: } \\
\text { 20\% de } \\
\text { diagnósticos }\end{array}$ & $78 \% \mathrm{M}$ & $\mathrm{Si}$ & $\begin{array}{l}\text { Primary Care: } 78 \% \\
\text { Pediatra: } 20 \% \\
\text { Neurologista: } 2 \% \\
\text { Psiquiatra Infantil: } \\
\text { não tem disponível } \\
\text { (sic-autor) }\end{array}$ \\
\hline $\begin{array}{l}\text { Fogelman, } \\
2003\end{array}$ & $\begin{array}{l}1999 \text { a 2001: } \\
21 \% \text { de } \\
\text { diagnósticos }\end{array}$ & $77 \% \mathrm{M}$ & $\mathrm{Si}$ & si \\
\hline $\begin{array}{l}\text { Huissoud, } \\
2002\end{array}$ & si & $85 \% \mathrm{M}$ & si & Pediatras: $65 \%$ \\
\hline
\end{tabular}

si: sem informação; +: alguma informação presente. 


\section{Sistema de prescrições}

$\mathrm{Na}$ França', os psicoestimulantes só podem ser iniciados por neurologistas, psiquiatras e pediatras que trabalham em hospitais. Médicos de família podem manter a prescrição sem modificação e durante só um ano, quando a pessoa terá que retornar ao especialista. Na Austrália², há necessidade de cadastro num departamento médico e usualmente são especialistas em pediatria, neurologia e psiquiatria (geral e infantil). Médicos generalistas podem manter as prescrições quando forem indicados pelo especialista cadastrado e quando forem autorizados. Segundo Calver ${ }^{3}$, $120 \mathrm{mg}$ de metilfenidato ou $2 \mathrm{mg} / \mathrm{kg} / \mathrm{dia}$ é a dose máxima permitida, acima da qual há exigência de explicações. Embora Carlini et al. ${ }^{4}$ relatem sobre o sistema restritivo das prescrições no Brasil, pode-se notar ${ }^{1-3}$ que há países mais restritivos. Na África do Sul5, todos os médicos podem prescrever. Huissoud et al. ${ }^{6}$ referem que na Suíça a prescrição do metilfenidato é controlada pelas autoridades, mas não detalham a forma. Não há comentários sobre o assunto em outros países.

\section{Sistemas de saúde}

Segundo Stevens?, nos atendimentos pelo Medicaid há mais diagnósticos de TDA/H e outros transtornos psiquiátricos, quando comparado com outros "sistemas ou planos de saúde" semelhantes ao Medicaid, que tem foco na atenção primária e no qual as pessoas afetadas são usualmente encaminhadas para especialistas. No caso de a pessoa já ter sido diagnosticada, prescrições de não estimulantes foram bem mais frequentes pelo sistema Medicaid que por planos privados.

\section{Tempo de uso}

Trutter ${ }^{5}$ referiu até 15 prescrições/ano e reconhece diminuição do uso no período das férias escolares. Castle et al. ${ }^{8}$ reportaram 227 tomadas/ano para menores de 10 anos e 200/ano no geral. Miller et al. ${ }^{9}$ destacaram que 33\% tiveram menos de 2 prescrições, 33\% tiveram prescrições por até 4 meses, 17\%, por um ano ou mais - mais comum entre meninos de até 8 anos e com primeira prescrição feita por psiquiatra (8,5\% dos prescritores versus 91,5\% de pediatras). Cox et al..$^{10}$ sugeriram média de 4 prescrições/ano e que 13\% tiveram somente uma receita. Knellwolf et al." mencionaram média global de 10,2 meses de uso para os iniciantes. O tempo de uso foi de curto prazo em $17 \%$, cinco meses em $34 \%$ e 26 meses em 50\%. Miller et al. ${ }^{12}$ acreditam que, com o tempo (a partir da 6a prescrição), aumenta a atuação dos generalistas e diminui a dos pediatras e psiquiatras.

\section{Aumento das prescrições}

Para Castle et al. ${ }^{8}$, entre 2000 e 2005 os tratamentos aumentaram 11,8\%; foram realizados mais em adultos (15,3\%) que em crianças (9,5\%); tiveram mais foco em mulheres de 20 a
44 anos (21,4\%); foram realizados mais nas meninas (13,3\%) que nos meninos (8,2\%). Em meninos houve maior aumento entre os de até 9 anos. Lin et al.13 afirmam que o aumento das prescrições de anfetaminas entre 1990 e 1997 foi de $817 \%$ para pessoas acima de 15 anos, $724 \%$ de 10 a 14 anos e 680\% de 5 a 9 anos. Segundo Miller et al.12, a prescrição de metilfenidato aumentou 5 vezes entre 1990 e 1996. Para McCarthy et al.14, entre 2000 e 2003 houve diminuição da administração de psicoestimulantes nas escolas com alunos de poder econômico mais elevado, sugerindo aumento do uso das medicações de longa ação, já que o mesmo não aconteceu com medicamentos "não controlados".

\section{Características socioculturais}

Stevens ${ }^{7}$ encontrou menos prescrições e acompanhamento médico em hispânicos em relação à população branca, questionando se isso se deu pela dificuldade de comunicação em inglês entre mães e médicos. Cox et al. ${ }^{10}$ ressaltaram que pessoas brancas foram 2 a 9 vezes mais medicadas quando comparadas com as de pele não branca, assim como pessoas que moram em regiões de mais poder econômico e famílias com menos de 4 crianças têm mais chance de receber prescrições. Miller et al.'2 encontraram leve superioridade de prescrições em grupos de poder econômico inferior. Em outro estudo ${ }^{14}, 89 \%$ das prescrições foram para pessoas de pele branca. Em Israel, Fogelman et al. ${ }^{15,16}$ concluíram que diferenças culturais geraram diferença na prescrição de até 30 vezes. Robison et al..$^{17}$ salientaram, também, que quase $90 \%$ das prescrições foram para pessoas brancas.

\section{Dosagens de metilfenidato}

Preen et al. ${ }^{2}$ e Miller et al..$^{12}$ enfatizaram que psiquiatras receitam doses maiores que pediatras, sendo que Preen et al. ${ }^{2}$ destacaram que os que usam 2 ou mais medicações também têm doses maiores de psicoestimulantes. Na África do Sul5, 50\% recebiam 10 mg/dia; 33\% - 20 mg/dia; e 7,8\% - acima de 30 mg/dia. Para Miller et al. ${ }^{2}$, a dosagem média para 5 dias úteis foi de $28,3 \mathrm{mg}$. Na Suíça, os autores ${ }^{6}$ encontraram média de $11,2 \mathrm{mg} /$ dia até 5 anos de idade e $24,5 \mathrm{mg} /$ dia para a faixa etária de 15 a 19 anos. Preen et al.18, em 2007, constataram que a dose média para todas as idades foi de $17,6 \mathrm{mg} / \mathrm{dia}, 8,6 \mathrm{mg} / \mathrm{dia}$ para 3 anos de idade e de $26,7 \mathrm{mg} /$ dia para os de 17 anos.

\section{Prescrição para idades de até 5 anos}

Preen et al. ${ }^{2}$ declararam prescrições para crianças entre 2 e 5 anos e Stevens ${ }^{7}$ para a partir de 3 anos. Huissoud ${ }^{6}$ revelou taxa de 0,2\% das prescrições para a população entre zero e 4 anos (só homens). Miller et al. ${ }^{9}$ realçaram que, entre 1990 e 1996, a média de prescrições para até 4 anos foi 7 versus média de 7,8 meses de tratamento para até 9 anos. Lin et al.13 afirmaram que entre 1990 e 1997 a prescrição de anfetaminas aumentou $380 \%$ para a faixa etária de 2 a 4 anos. Calver et al. ${ }^{3}$ 
e Preen et al. ${ }^{18}$ reportaram, respectivamente, 3\% das prescrições para a faixa etária de 2 a 5 anos e para 3 a 5 anos.

\section{Resultados comuns}

Aumentos graduais das prescrições de psicoestimulantes têm o sexo masculino como o maior alvo; baixo percentual de pessoas medicadas proporcionalmente à prevalência conhecida; pediatras e generalistas são os maiores prescritores; maior frequência na população de 7 a 15 anos e progressivo aumento das medicações de ação prolongada (Quadro 2).

\section{DISCUSSÃO}

Os resultados serão tratados em 3 blocos: resultados esperados, itens relevantes e questões preocupantes.

Os resultados esperados foram: o aumento geral das prescrições e dos diagnósticos e gradativo das medicações de liberação prolongada; os homens e as crianças são o alvo da maioria; o sistema de saúde influencia nos diagnósticos e nas prescrições; os quadros mais complexos estão sob a assistência do psiquiatra, apesar de não serem os "maiores" prescritores.

O aumento dos diagnósticos e prescrições de psicoestimulantes é um fato positivo, já que demonstra mais atenção do médico quanto ao TDA/H e aos estimulantes e, portanto, menos receio ao seu receituário, como era visto há algumas décadas. Somente um artigo² faz essa referência.

Pressupondo-se que o TDA/H seja de origem neurobiológica e de curso crônico, o esperado é que a medicação seja mantida por anos, mas o encontrado foi que em até $50 \%$ das pessoas ela não foi mantida por mais de 5 meses ${ }^{9-11}$, verificando-se baixos níveis de adesão. Isso leva a pensar que os médicos estão falhando no aspecto técnico (diagnóstico) ou na questão cultural (interlocução com a família).

O gradativo aumento das prescrições dos estimulantes de longa ação ${ }^{14}$ também já é percebido clinicamente no Brasil, assim como sua relação com o poder econômico da família.

A questão de os homens serem o alvo mais frequente das prescrições (Quadro 2) pode ser explicada pelo fato de que são eles mais afetados pela forma hiperativa, que é mais aparente e incomoda mais. Talvez a cultura gere a crença de normalidade para as meninas serem quietas desatentas. Felizmente, tal questão parece estar mudando, como referem Castle et al. ${ }^{8}$ em 2007.

Outra informação que não se constituiu novidade é que a maioria das prescrições foi dada para crianças entre 6 e 14 anos, embora estejam aumentando nas idades adultas ${ }^{8}$. Esse resultado pode estar demonstrando a real preocupação das famílias com o bom desenvolvimento escolar dos filhos e uma melhor qualidade de vida para os adultos afetados.

A influência do sistema de saúde na qualidade técnica (diagnósticos e prescrições) é fato de alerta, pois, como nos EUA $^{7}$, pode também estar acontecendo no Brasil.
A questão de que a maioria das prescrições (Quadro 2) tem origem em especialidades diferentes da psiquiatria não é relevante, pois, assim como é citado em artigos clínicos 2,12, esta revisão também mostra que os quadros mais complexos (que necessitam de doses maiores e/ou de mais de um tipo de medicação) estão sob a assistência do psiquiatra, o que é esperado.

Os itens relevantes foram: a influência do aspecto sociocultural e as baixas dosagens utilizadas, exceto nos Estados Unidos.

A influência da condição sociocultural, exceção a Miller et al. ${ }^{12}$, que pode ser quantificada de "absurda" e só foi relatada em trabalhos dos EUA $7,10,14$ e de Israel"16,17, é importante, pois demonstra que os médicos falham em questões que deveriam ser técnicas, não valorizando as queixas dos familiares ou não conseguindo equacionar relatos (queixas) de familiares/ professores naquele meio social em que o paciente vive, que é um meio "estranho" ao médico. A maior importância disso é que também no Brasil existe tal discrepância do poder econômico e da cultura - estaria isso ocorrendo com nossa população também? E o lado perverso é que esse segmento econômico-cultural da população, se afetado pelo TDA/H, poderia ser muito favorecido com os tratamentos adequados.

As dosagens referidas (exceto nos Estados Unidos) parecem ser baixas, uma vez que na África do Sul, em artigo que atingiu pessoas com até 25 anos, mas com idade média de 10,5 anos (SD 3,9), a autora ${ }^{5}$ ressaltou trabalho em que 50\% recebiam 10 mg/dia; 33\% - 20 mg/dia; e 7,8\% acima de 30 mg/dia. E na Suíça os autores ${ }^{6}$ encontraram média de 11,2 mg/dia até 5 anos de idade e 24,5 mg/dia para a faixa de 15 a 19 anos. Se se analisarem tais valores com dados do Brasil, Sociedade Brasileira de Pediatria ${ }^{20}$, mesmo sabendo que nossa população é mais baixa e menos pesada, as doses médias encontram-se abaixo de 0,5 mg/kg/dia (peso de 33 kg para meninos com 10,5 anos, de 56,7 kg para 15 anos e de 68,9 kg para 18 anos).

Duas questões são preocupantes: a baixa adesão ao tratamento medicamentoso e a sua utilização em crianças muito novas - 2 anos!

O tempo de uso dos medicamentos parece muito baixo. Têm-se 54 semanas e aproximadamente 260 dias úteis no ano, descontados os feriados. Comparando tais dados com os de Castle et al. ${ }^{8}$, deduz-se que para o resultado geral há 4 tomadas por semana.

Os resultados encontrados pelos autores americanos, 90 mostram-se similares aos encontrados pelos franceses ${ }^{11}$ : aproximadamente 50\% têm prescrições por curto prazo (até 5 meses), e isso reforça a hipótese de falha técnica (diagnóstico) ou da influência de crenças pelas quais a família não aceita o diagnóstico, ou não fica satisfeita com a forma como o diagnóstico foi apresentado ou mesmo tem receio de me- 
dicar o/a filho/a. A hipótese restante, de que 50\% das pessoas apresentam reações adversas intensas a ponto de pararem de usar a medicação, é pouco provável estatisticamente se o processo diagnóstico seguiu as normas técnicas.

Não há evidências científicas para o uso de psicoestimulantes em crianças tão novas (até 4 anos) quanto as encontradas na revisão. O que está acontecendo com os familiares e professores para essa demanda? Uma resposta hipotética é que, como as famílias estão progressivamente menores (menos filhos - crianças), com mais mobilidade de parceiros e geográfica e jornadas duplas de trabalho, as pessoas estão ficando mais intolerantes com a normal inquietação motora das crianças dessa faixa etária. Tal hipótese é fundamentada pelo encontrado por Cox et al. ${ }^{10}$, que em famílias com mais crianças há menos prescrições de estimulantes. E, pelo viés do profissional médico, há demanda técnica real para a medicalização de até 3\% dessa população ${ }^{3,18}$ ou está havendo apenas respostas reativas às demandas?

\section{CONCLUSÃO}

Este trabalho possibilita responder 3 das 5 perguntas formuladas nos objetivos: não é o psiquiatra o "maior" prescritor de psicoestimulante e muito menos, portanto, o subespecialista da infância e adolescência; 50\% das pessoas usam tais medicações por menos de um semestre; e é o psiquiatra quem acompanha quadros mais complexos.

A pergunta sobre se a realidade encontrada na revisão é similar à nossa traz 2 respostas: é quase certo que todos os resultados categorizados no bloco de resultados esperados também ocorrem no Brasil, mas os outros itens têm que ser objeto de pesquisa nacional para que as respostas tenham fundamentação.

A baixa adesão ao tratamento do TDA/H merece várias pesquisas sobre pelo menos 4 pontos: do curso crônico no universo infantil, das crenças dos familiares ante os filhos crianças - afetados por um transtorno psiquiátrico, da aceitação de tratamento medicamentoso por longo prazo para o/a filho/a em conexão com as questões técnicas específicas sobre a cronicidade do TDA/H. Os trabalhos devem também comparar o TDA/H com outros transtornos crônicos da psiquiatria geral e infantil, assim como com transtornos crônicos não psiquiátricos, para buscar os procedimentos clínicos pragmáticos que efetivamente melhorem a qualidade assistencial de nossa população afetada. Uma via para se alcançar esse objetivo foi concretizada pela Resolução RDC n²7, de 30 de março de 2007, da Agência Nacional de Vigilância Sanitária (ANVISA) ${ }^{21}$, que centraliza as informações das prescrições de talonário tipo "A". Também a partir dessas informações será possível saber se as dosagens prescritas são ou não as recomendadas nos manuais clínicos.
Seria muito saudável para todos se o alto percentual de prescrições de psicoestimulantes para crianças com menos de 4/5 anos não ocorresse no Brasil até que evidências científicas as corroborassem. Tal situação poderia ser evitada se as instituições formadoras de opinião incluíssem esse item nos programas de educação médica continuada.

\section{REFERÊNCIAS}

1. Frances C, Hoizey G, Nillart H, Trenque T. Pediatric and methylphenidate (Ritalin) restrictive conditions of prescription in France. Br J Clin Pharmacol. 2004;57(1):115-6.

2. Preen DB, Calver J, Sanfilippo FM, Bulsara M, Holman CD. Prescribing of psychostimulants medications for attention-deficit hyperactivity disorder in children: differences between clinical specialities. Med J Aust. 2008;188(6):337-9.

3. Calver J, Sanfilippo F, Preen D, Bulsara M. Prescribed stimulants use by Western Australians with attention-deficit hyperactivity disorder (ADHD): does amount dispensed exceed the expected authorised use? Aust N Z Public Health. 2007;31(6):533-9.

4. Carlini EA, Nappo SA, Nogueira V, Naylor FGM. Metilfenidato: influência da notificação de receita $A$ (cor amarela) sobre a prática de prescrição por médicos brasileiros. Rev Psiq Clin. 2003; 30(1):11-20.

5. Trutter I. Methylphenidate: prescribing patterns in South African primary care patient population. J Clin Pharm Ther. 2005;30(1):59-63

6. Huissoud T, Jennin F, Dubois-Arber F. Hyperactivité et prescription de Ritaline dans le canton de Vaud (Suisse). Rev Epidemiol Sante Publique. 2007;55(5):357-63.

7. Stevens J. Ethnic and regional differences in primary care visits for attention-deficit hyperactivity disorder. J Dev Behav Pediatr. 2004;25(5):318-25.

8. Castle L, Aubert RE, Verbrugge RR, Khalid M, Epstein RS. Trends in medication treatment for ADHD. J Atten Disord. 2007;10(4):335-42.

9. Miller AR, Lalonde CE, McGrail KM. Children's persistence with methylphenidate therapy: a population-based study. Can J Psychiatry. 2004;49(11):761-8.

10. Cox ER, Motheral BR, Henderson RR, Mager D. Geographic variation in the prevalence of stimulant medication use among children 5 to 14 years old: results from a commercially insured US sample. Pediatrics. 2003;111:237-43.

11. Knellwolf A-L, Deligne J, Chiarotti F, Auleley GR, Palmieri S, Boisgrad CB, et al. Prevalence and patterns of methylphenidate use in French children and adolescents. Eur J Clin Pharmacol. 2008;64:311-7.

12. Miller AR, Lalonde CE, McGrail KM, Armstrong RW. Prescription of methylphenidate to children and youth, 1990-1996. (AMJ. 2001;165(11):1489-94.

13. Lin SJ, Crawford SY, Lurvey PL. Trend and variation in amphetamine prescription usage among children and adolescents in Michigan. Soc Sci Med. 2005;60(3):617-25.

14. McCarthy AM, Kelly MW, Johnson S, Roman J, Zimmerman MB. Changes in medications administered in schools. J Sch Nurs. 2006;22(2):102-7.

15. Fogelman Y, Kahan E. Methylphenidate use for attention-deficit hyperactivity disorder in northern Israel - a controversial issue. Isr Med Assoc J. 2001;3(12):925-7.

16. Fogelman $Y$, Vinker $S$, Guy N, Kahan E. Prevalence and change in the prescription of methylphenidate in Israel over 2-year period. CNS Drugs. 2003;17(12):915-9.

17. Robison LM, Sclar DA, Skaer TL, Galin RS. National trends in the prevalence of attentiondeficit/hyperactivity disorder and the prescribing of methylphenidate among scholl-age children: 1990-1995. Clin Pediatr (Phila). 1999;38(4):209-17.

18. Preen DB, Calver J, Sanfilippo FM, Bulsara M, Holman CDJ. Aust N Z J Public Health. 2007:31:120-6

19. Rees A. Ritalin use in BC. CMAJ. 2000;162(6):753-4.

20. Sociedade Brasileira de Pediatria. Valores de referência. [acesso em 24 fev 2009] Disponível em: http: //www.sbp.com.br/img/documentos/valores_referencia.pdf.

21. Agência Nacional de Vigilância Sanitária. Legislação em Vigilância Sanitária. [acesso em 25 fev 2009] Disponível em: http://e-legis.anvisa.gov.br/leisref/public/showAct.php. 